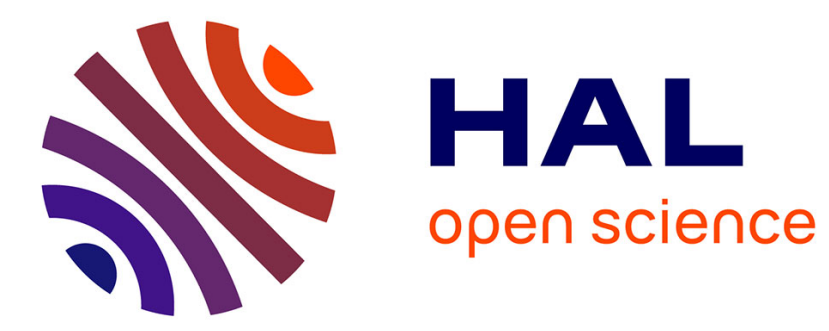

\title{
Observability and Detectability of Singular Linear Systems with Unknown Inputs
}

Francisco Javier Bejarano, Thierry Floquet, Wilfrid Perruquetti, Gang Zheng

\section{To cite this version:}

Francisco Javier Bejarano, Thierry Floquet, Wilfrid Perruquetti, Gang Zheng. Observability and Detectability of Singular Linear Systems with Unknown Inputs. Automatica, 2013, 49 (3), pp.6. 10.1016/j.automatica.2012.11.043 . hal-00753706

\section{HAL Id: hal-00753706 https://inria.hal.science/hal-00753706}

Submitted on 8 Mar 2017

HAL is a multi-disciplinary open access archive for the deposit and dissemination of scientific research documents, whether they are published or not. The documents may come from teaching and research institutions in France or abroad, or from public or private research centers.
L'archive ouverte pluridisciplinaire HAL, est destinée au dépôt et à la diffusion de documents scientifiques de niveau recherche, publiés ou non, émanant des établissements d'enseignement et de recherche français ou étrangers, des laboratoires publics ou privés. 


\title{
Observability and Detectability of Singular Linear Systems with Unknown Inputs ${ }^{\text {th }}$
}

\author{
Francisco Javier Bejarano ${ }^{\mathrm{a}}$, Thierry Floquet ${ }^{\mathrm{a}, \mathrm{b}}$, Wilfrid Perruquetti ${ }^{\mathrm{a}, \mathrm{b}}$, Gang Zheng ${ }^{\mathrm{a}}$ \\ ${ }^{a}$ Non-A, INRIA - Lille Nord Europe, 40 avenue Halley, Villeneuve d'Ascq 59650 , France. \\ ${ }^{b}$ LAGIS FRE CNRS 3303, Villeneuve d'Ascq 59651, France.
}

\begin{abstract}
In this paper the strong observability and strong detectability of a general class of singular linear systems with unknown inputs are tackled. The case when the matrix pencil is non-regular is comprised (i.e., more than one solution for the differential equation is allowed). It is shown that, under suitable assumptions, the original problem can be studied by means of a regular (non-singular) linear system with unknown inputs and algebraic constraints. Thus, it is shown that for purposes of analysis, the algebraic equations can be included as part of an extended system output. Based on this analysis, we obtain necessary and sufficient conditions guaranteing the observability (or detectability) of the system in terms of the zeros of the system matrix. Corresponding algebraic conditions are given in order to test the observability and detectability. A formula is provided that expresses the state as high order derivative of a function of the output, which allows for the reconstruction of the actual state vector. It is shown that the unknown inputs may be reconstructed also.
\end{abstract}

Keywords: Singular systems, strong detectability, strong observability, algebraic observability.

\section{Introduction}

The problem of designing an observer for a multi-variable linear system partially driven by unknown inputs (UI) has been widely studied (Darouach et al. (1994); Floquet and Barbot (2004); Floquet et al. (2007); Guan and Saif (1992)). Such observers can be of important use for systems subject to disturbances or with inaccessible inputs, or when dealing with the problem of fault diagnosis. Observability and the problem of observer design have been widely studied for singular systems with perfectly known model. The problems of solvability, controllability and observability were studied in Yip and Sincovec (1981). There, the observability analysis is addressed and algebraic characterizations were found. By using the distributional framework, in Cobb (1984) the algebraic duality between controllability and observability is proven. Necessary and sufficient conditions allowing for the design of a Luenbergerlike observer were found in Paraskevopoulos and Koumboulis (1992). In the three previous mentioned works, it was considered that the system has a regular matrix pencil which entails a unique state solution. Without any particular assumption over the matrix pencil of the system, the casual observability, which does not allow to use neither the derivatives of the input nor the derivatives of the output, is studied in Hou and Müller (1999a). The same authors suggest an observer design in Hou and Müller (1999b). In that work it is shown that by allowing the derivatives of the input and output to be involved in the

\footnotetext{
${ }^{2}$ Corresponding author F.J. Bejarano, he held a posdoctoral position with the ALIEN team.

Email addresses: javbejarano@yahoo.com.mx (Francisco Javier Bejarano), thierry.floquet@ec-lille.fr (Thierry Floquet), wilfrid.perruquetti@inria.fr (Wilfrid Perruquetti), gang.zheng@inria.fr (Gang Zheng)
}

observer (called it there as a generalized observer), detectability is enough for the convergence of the observation error. A reduced order observer is designed in Darouach and Boutayeb (1995). In Darouach and Boutat-Baddas (2008) an observer for nonlinear singular systems is proposed. In spite of the extended literature regarding the observability analysis and synthesis of singular systems, there exist few results dealing with such problems when the system contains UI. In Paraskevopoulos et al. (1992), the observer design problem is considered for singular linear systems with UI (SLSUI) and necessary and sufficient conditions are given for the design of a Luenberger-like observer. In Darouach et al. (1996), a reduced order observer is proposed. Under some regularity conditions, the observer design is studied in Chu and Mehrmann (1999). Meanwhile, in Koenig (2005) a proportional multiple-integral observer is proposed. Using the graph-theory approach, observability conditions are found in Boukhobza and Hamelin (2007).

In this note, the observability problem of a general class of singular linear systems with UI is studied. The system is not required to have a regular matrix pencil. We obtain necessary and sufficient conditions for the strong observability and strong detectability. We show that the reconstruction of the state can be carried out by a formula that expresses the actual state as a high order derivative of a function of the output. The manuscript also includes Section 2, where the system is described and the strong observability and strong detectability are defined. In Section 3 , we obtain the necessary and sufficient conditions which allow for the reconstruction of the state vector. Section 4 deals with an explicit formula that allows for the reconstruction of the state vector. The finite time reconstruction (observability) is considered in 4.1. The procedure for the asymptotic reconstruction (detectability) is given in 4.2. A summarized algo- 
rithm explaining all the estimation procedure is presented in 4.3. To reenforce the theoretical results, we present an example with simulations in Section 5. The following notation will be used throughout the paper. For a matrix $X$, we denote by $X^{\perp}$ a full row rank matrix such that $X^{\perp} X=0$, and by $X^{\perp \perp}$ a full row rank matrix such that $\operatorname{rank} X^{\perp \perp} X=\operatorname{rank} X$ (then the ma$\operatorname{trix}\left[\begin{array}{ll}\left(X^{\perp}\right)^{T} & \left(X^{\perp \perp}\right)^{T}\end{array}\right]^{T}$ is nonsingular). The Moore-Penrose pseudo-inverse matrix of $X$ is denoted by $X^{+}$. The rank of $X$ is denoted by means of $\rho_{X}$. By $\|\cdot\|$, we mean the Euclidean norm. $\mathbb{C}^{-}$denotes de set of complex numbers with strictly negative real part. $I_{r}$ is the identity matrix of dimension $r$ by $r .0_{r \times s}$ is the zero matrix of dimension $r$ by $s$. For the limit from above, $x\left(0^{+}\right)=\lim _{t \rightarrow 0^{+}} x(t)$.

\section{System Description and Problem Formulation}

Let us consider the SLSUI governed by the following equations

$$
\Sigma:\left\{\begin{array}{rl}
E \dot{x}(t) & =A x(t)+D \mu(t) \\
y(t) & =C x+F \mu(t)
\end{array},\right.
$$

where $x(t) \in \mathbb{R}^{n}$ is the state vector, $y(t) \in \mathbb{R}^{p}$ is the system output, and $\mu(t) \in \mathbb{R}^{m}$ is the unknown input vector. Matrices $E, A \in \mathbb{R}^{n \times n}, D \in \mathbb{R}^{n \times m}, C \in \mathbb{R}^{p \times n}$, and $F \in \mathbb{R}^{p \times m}$ are all constant. The matrix $E$ is assumed to be singular. Given a state $x_{0} \in \mathbb{R}^{n}$ and a function $\mu(t)$, we denote by $x_{\mu}\left(x_{0}, t\right)$ the state of $\Sigma$ at time $t$ which results from taking the initial condition equal to $x_{0}$ and the input vector is equal to $\mu$. Therefrom, in a straightforward manner we define the output $y_{\mu}\left(x_{0}, t\right)=C x_{\mu}\left(x_{0}, t\right)+F \mu(t)$.

We are interested in the reconstruction of the (non-impulsive) trajectory of state vector $x(t)$ given the output information $y(\tau)_{\tau \in[0, t]}$. System $\Sigma$ is not assumed to have a regular pencil (Kaczorek (2007)), i.e., it is allowed that $\operatorname{det}(s E-A)=0$ for all $s \in \mathbb{C}$ (then $x_{\mu}\left(x_{0}, t\right)$ may have more than a solution). Nevertheless, $\mu(t)$ must be so that $x(t)$ be piecewise continuous for all $t>0$; however, an impulse may occur at $t=0$. In order to give algebraic conditions allowing the reconstruction of $x(t)$, we consider the following definitions, which are based on classical definitions for linear time invariant systems (see, e.g. Trentelman et al. (2001)).

Definition 1 (Strong observability). The system $\Sigma$ is strongly observable (SO) if for all $x_{0} \in \mathbb{R}^{n}$ and for every input function $\mu$, the following implication is satisfied

$$
y_{\mu}\left(x_{0}, t\right)=0 \forall t>0 \text { implies } x\left(0^{+}\right)=0 .
$$

Definition 2 (Strong detectability). The system $\Sigma$ is strongly detectable (SD) if for all $x_{0} \in \mathbb{R}^{n}$ and for every input function $\mu$, the following implication holds

$$
y_{\mu}\left(x_{0}, t\right)=0 \forall t>0 \text { implies } \lim _{t \rightarrow \infty} x_{\mu}\left(x_{0}, t\right)=0 .
$$

It is clear that strong observability is a necessary condition to reconstruct the entire trajectory of the state $x(t)$. Indeed, let us suppose that $\Sigma$ is not strongly observable, then it means that there exist $\bar{\mu}$ and $\bar{x}_{0}$ such that $y_{\bar{\mu}}\left(\bar{x}_{0}, t\right)=0 \forall t>0$, but $x\left(0^{+}\right) \neq 0$. Then, since we assume that $x(t)$ is piecewise continuous, then $x_{\mu=0}(0, t) \equiv 0$ and $x_{\bar{\mu}}\left(\bar{x}_{0}, t\right) \neq 0$ in an open interval, however, both yield a system output identically equal to zero. Thereby, it would be impossible to reconstruct the entire state trajectory. Below it would be proven that SO is a structural necessary and sufficient condition for the reconstruction in finite time of $x(t)$. Analogously, it will be shown that SD is a structural necessary and sufficient condition for the asymptotic reconstruction of $x(t)$.

\section{Observability Analysis}

Since $E$ is singular, there exist non-singular matrices $T \in$ $\mathbb{R}^{n \times n}$ and $S \in \mathbb{R}^{n \times n}$ such that $E$ can be transformed as follows ${ }^{1}$

$$
T E S=\left[\begin{array}{cc}
I_{\rho_{E}} & 0 \\
0 & 0
\end{array}\right]
$$

Thus, let us define the vector $z:=\left[\begin{array}{ll}z_{1}^{T} & z_{2}^{T}\end{array}\right]^{T}=S^{-1} x$, where $z_{1} \in \mathbb{R}^{\rho_{E}}$ and $z_{2} \in \mathbb{R}^{n-\rho_{E}}$. In these new coordinates, $\Sigma$ can be rewritten as follows

$$
\Psi:\left\{\begin{array}{rl}
T E S \dot{z}(t) & =T A S z(t)+T D \mu(t) \\
y(t) & =C S z(t)+F \mu(t)
\end{array} .\right.
$$

In view of (4), $\Psi$ takes the following form

$$
\begin{aligned}
\dot{z}_{1}(t) & =T_{1} A S_{1} z_{1}(t)+T_{1} A S_{2} z_{2}(t)+T_{1} D \mu(t), \\
0 & =T_{2} A S_{1} z_{1}(t)+T_{2} A S_{2} z_{2}(t)+T_{2} D \mu(t), \\
y & =C S_{1} z_{1}(t)+C S_{2} z_{2}(t)+F \mu(t) .
\end{aligned}
$$

where $S_{1}$ and $S_{2}$ matrices arise from the following partition of $S, S=\left[\begin{array}{ll}S_{1} & S_{2}\end{array}\right]$ with $S_{1} \in \mathbb{R}^{\rho_{E} \times \rho_{E}}$ and $S_{2} \in \mathbb{R}^{\rho_{E} \times n-\rho_{E}}$. Analogously, $T_{1}$ and $T_{2}$ matrices come from the partition $T^{T}=$ $\left[\begin{array}{cc}T_{1}^{T} & T_{2}^{T}\end{array}\right]$ with $T_{1} \in \mathbb{R}^{\rho_{E} \times n}$ and $T_{2} \in \mathbb{R}^{n-\rho_{E} \times n}$. It is clear that $\Sigma$ is SO (resp. SD) ${ }^{2}$ if, and only if, $\Psi$ is SO (resp. SD). Below we will see that a simple manner to study the observability of $\Psi$, and by extension of $\Sigma$, is by considering (6b) as part of the system output of a new pseudo system and considering $z_{2}$ as part of the vector of UI. Indeed, let us define the system $\Phi$ by means of the following equation,

$$
\Phi:\left\{\begin{array}{rl}
\dot{z}_{1}(t) & =\bar{A} z_{1}(t)+\bar{D} v(t) \\
\bar{y}(t) & =\bar{C} z_{1}(t)+\bar{F} v(t)
\end{array},\right.
$$

where $v(t) \in \mathbb{R}^{n-\rho_{E}+m}, \bar{y}(t) \in \mathbb{R}^{n-\rho_{E}+p}$ and the matrices $\bar{A}, \bar{D}, \bar{C}$, and $\bar{F}$ are defined as follows

$$
\begin{gathered}
\bar{A}=T_{1} A S_{1}, \quad \bar{D}=\left[\begin{array}{cc}
T_{1} A S_{2} & T_{1} D
\end{array}\right], \\
\bar{C}=\left[\begin{array}{c}
T_{2} A S_{1} \\
C S_{1}
\end{array}\right], \quad \bar{F}=\left[\begin{array}{cc}
T_{2} A S_{2} & T_{2} D \\
C S_{2} & F
\end{array}\right] .
\end{gathered}
$$

\footnotetext{
${ }^{1}$ We might select $S=\left[\begin{array}{ll}S_{1} & S_{2}\end{array}\right]$ to be non-singular and so that $\operatorname{im} S_{2}=\operatorname{ker} E$. Thus, $E S=\left[\begin{array}{cc}E S_{1} & 0\end{array}\right]$ and $\operatorname{rank} E S_{1}=\operatorname{rank} E$. Then a non-singular matrix $T$ might be selected as $T^{T}=\left[\begin{array}{cc}T_{1}^{T} & T_{2}^{T}\end{array}\right]$ so that $T_{1} E S_{1}=I$ and $T_{2} E S_{1}=0$, one possibility is to select $T_{1}=\left(E S_{1}\right)^{+}=$ $\left[\left(E S_{1}\right)^{T}\left(E S_{1}\right)\right]^{-1}\left(E S_{1}\right)^{T}$.

${ }^{2}$ Respectively strongly detectable.
} 
It is clear by (6) that $\Phi$ looks like system $\Psi$. In general, they do not represent identical systems. However, both systems are identical if these both identities hold: $\bar{y}^{T}=\left[\begin{array}{cc}0^{T} & y^{T}\end{array}\right]$ and $v^{T}(t)=\left[\begin{array}{ll}z_{2}^{T}(t) & \mu^{T}(t)\end{array}\right]$. In the next theorem it is claimed that the fulfillment of the SO (resp. SD) of $\Sigma$ is equivalent to the fulfillment of the SO (resp. SD) of $\Phi$ (condition needed for the reconstruction of $z_{1}$ ) plus a rank condition (required for the reconstruction of $z_{2}$ ).

Theorem 1. System $\Sigma$ is $S O$ (resp. SD) if, and only if, $\Phi$ is $S O$ (resp. SD) and the following rank condition holds

$$
\rho_{\bar{B}}=n-\rho_{E}+\operatorname{rank}\left[\begin{array}{c}
D \\
F
\end{array}\right] \text {, where } \bar{B}:=\left[\begin{array}{c}
\bar{D} \\
\bar{F}
\end{array}\right] .
$$

Furthermore, this equivalence is independent of the choice of $T$ and $S$.

Proof. Firstly, notice that, since $\operatorname{rank}\left[\begin{array}{cc}D^{T} & F^{T}\end{array}\right]=$ $\operatorname{rank}\left[\begin{array}{ll}(T D)^{T} & F^{T}\end{array}\right]$, the fulfillment of (9) is equivalent to say that $\bar{B}\left[\begin{array}{ll}z_{2}^{T} & \mu^{T}\end{array}\right]^{T}=0$ only if $z_{2}=0$.

Necessity. Let $\Sigma$ be SO (resp. SD). Let us suppose that, for an input $v$ and a state $z_{1}, \bar{y}_{v}\left(z_{1}, t\right)=0$. For the case when $z_{2}(t)$

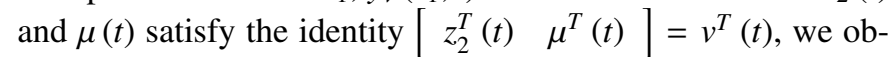
tain that $\Psi$ and $\Phi$ represent the same system. Thus, defining $x_{0}=S z(0)$ we conclude that $y_{\mu}\left(x_{0}, t\right)=0$ for all $t>0$. Now, since, by assumption, (2) (resp. (3)) holds, $x\left(0^{+}\right)=0$ (resp. $x(t)$ converges to zero), which in turn implies that $z\left(0^{+}\right)=0$ (resp. $z(t)$ converges to zero), in particular $z_{1}\left(0^{+}\right)=0$ (resp. $\lim _{t \rightarrow \infty} z_{1}(t)=0$ ), i.e. $\Phi$ is SO (resp. SD).

Now, assume that (9) does not hold. Then, there exists a vector $v$ which can be divided as $v^{T}=\left[\begin{array}{cc}v_{1}^{T} & v_{2}^{T}\end{array}\right]\left(v_{1} \in \mathbb{R}^{n-\rho_{E}}\right.$, $\left.v_{2} \in \mathbb{R}^{m}\right)$ so that $\bar{B} v=0$ and $v_{1} \neq 0$. By selecting $z_{2}(t)=v_{1}$ and $\mu(t)=v_{2}$, and $z_{1}\left(0^{+}\right)=0$, eq. (6) is fulfilled and $y(t)=0$ for all $t>0$. Therefore $x(t)=S z(t)=S\left[\begin{array}{cc}0 & v_{1}^{T}\end{array}\right]^{T}=$ const $\neq 0$. That is, in such a case $\Sigma$ is not SO (resp. $\Sigma$ is not SD).

Sufficiency. Firstly, let us notice that $y_{\mu}\left(x_{0}, t\right) \equiv 0$ implies $\bar{y}_{v}\left(z_{10}, t\right) \equiv 0$ for some $z_{10}$ and $v$. Indeed, let us suppose that $y_{\mu}\left(x_{0}, t\right)=0$ for a state $x_{0} \in \mathbb{R}^{n}$ and an input function $\mu$. Then, the function $z_{\mu}\left(z_{0}, t\right)=S^{-1} x_{\mu}\left(x_{0}, t\right)$ satisfies (6), specifically the algebraic constraint in (6b) is fulfilled. Therefore, we conclude that, for system $\Phi, \bar{y}_{v}\left(z_{10}, t\right)=0$, for all $t>0$, with $v$ as the extended vector of $z_{2}$ and $\mu$. Furthermore, it is known, from linear system theory, that an input $v(t)$ zeroing the output must have the form $v(t)=K^{*} z_{1}(t)+L w(t)(t>0)$, for a particular matrix $K^{*}$, a matrix $L$ such that $\bar{B} L=0$, and a function $w(t)$.

Thus, assuming that $\Phi$ is SO (resp SD), $y_{\mu}\left(x_{0}, t\right)=0$ for all $t>0$, implies, since also $\bar{y}_{v}\left(z_{10}, t\right)=0$, for all $t>0$, that $z_{1}(t) \equiv 0$ (resp. $z_{1}(t)$ converges to zero) and $\bar{B} v=0$ (resp. $\left.\bar{B} v=K^{*} z_{1}(t)\right)$. The previous identity, assuming that (9) is true, implies that $z_{2}(t) \equiv 0$ (resp. $z_{2}(t)$ converges to zero). Therefore, in such a case, $z(t) \equiv 0$ and so $x(t) \equiv 0$. Therefore, we conclude that $\Sigma$ is SO (resp. SD).

The independence from $T$ and $S$ is straightforward since we have proven Theorem 1 for arbitrary $T$ and $S$ satisfying (4).
As for $\Sigma$, we could expect that SO and SD can be completely characterized by the five-tuple $(E, A, C, D, F)$. Indeed, let $R(s)$ be the so-called system matrix of $\Sigma$, i.e.,

$$
R(s)=\left[\begin{array}{cc}
s E-A & -D \\
C & F
\end{array}\right], s \in \mathbb{C} .
$$

We say that $s_{0} \in \mathbb{C}$ is a zero of $\Sigma$ if $\rho_{R\left(s_{0}\right)}<n+\operatorname{rank}\left[\begin{array}{l}D \\ F\end{array}\right]$. Let $\sigma_{z}(\Sigma)$ be defined as the set of zeros of $\Sigma$. Let us characterize $\mathrm{SO}$ and SD in terms of the zeros of $\Sigma$.

Corollary 2. System $\Sigma$ is $S O$ (resp. SD) if, and only if, $\sigma_{z}(\Sigma)=$ $\varnothing\left(\right.$ resp. $\left.\sigma_{z}(\Sigma) \subset \mathbb{C}^{-}\right)$.

Proof. Let us define $Q(s)=\left[\begin{array}{cc}s I-\bar{A} & -\bar{D} \\ \bar{C} & \bar{F}\end{array}\right]$, which is the system matrix of $\Phi$. In view of (4) and (8), we obtain that,

$$
\left[\begin{array}{ll}
T & 0 \\
0 & I
\end{array}\right] R(s)\left[\begin{array}{cc}
S & 0 \\
0 & I
\end{array}\right]=Q(s)
$$

Thus, we deduce that $\rho_{R(s)}=\rho_{Q(s)}$. Then, Corollary 2 follows from the claims of Theorem 1 and the fact that $\Phi$ is SO (resp. SD) if, and only if, $\sigma_{z}(\Phi)=\varnothing\left(\operatorname{resp} . \sigma_{z}(\Phi) \subset \mathbb{C}^{-}\right)^{3}$.

\section{Algebraic Observability}

As we might expect SO coincides with algebraic observability (AO): we say that $\Sigma$ is AO if $x$ can be expressed as an algebraic function of $y$ and a finite number of its derivatives (see, e.g. Diop and Fliess (1991)). Let $M_{k}(k \geq 1)$ be the matrices obtained by the following recursive algorithm (see, Molinari (1976)),

$$
\begin{aligned}
M_{k+1} & =N_{k+1}^{\perp \perp} N_{k+1}, \quad M_{1}=\left(\bar{F}^{\perp} \bar{C}\right)^{\perp \perp} \bar{F}^{\perp} \bar{C}, \\
N_{k+1} & =T_{k}\left(\begin{array}{c}
M_{k} \bar{A} \\
\bar{C}
\end{array}\right), T_{k}=\left(\begin{array}{c}
M_{k} \bar{D} \\
\bar{F}
\end{array}\right)^{\perp} .
\end{aligned}
$$

Let us denote by $l$, the smallest integer such that $\rho_{M_{l}}=\rho_{M_{l+1}}$. For our purposes, we point out that $\Phi$ is SO if, and only if, $\rho_{M_{l}}=\rho_{E}$. For the case of SD we have to work a bit more with system $\Phi$. Indeed, let us assume that $\rho_{M_{l}}<\rho_{E}$. Let $V$ be a full column rank matrix so that $M_{l} V=0$. There exists a pair of matrices $Q$ and $K^{*}$ such that

$$
\bar{A} V+\bar{D} K^{*}=V Q \text { and } \bar{C} V+\bar{F} K^{*}=0 .
$$

From (12), it is clear that the $\operatorname{im}\left(\left(\bar{A}+\bar{D} K^{*} V^{+}\right) V\right) \subset \operatorname{im} V$ and $\left(\bar{C}+\bar{F} K^{*} V^{+}\right) V=0$. We can define a non-singular matrix $P$ of dimension $\rho_{E}$ as $P=\left[\begin{array}{c}M_{l} \\ V^{+}\end{array}\right], \quad P^{-1}=\left[\begin{array}{cc}M_{l}^{+} & V\end{array}\right]$, where $V^{+}$and $M_{l}^{+}$are the Moore-Penrose pseudo-inverse of $V$ and $M$, respectively: $V^{+}=\left(V^{T} V\right)^{-1} V^{T}$ and $M_{l}^{+}=M_{l}^{T}\left(M_{l} M_{l}^{T}\right)^{-1}$. By defining the vectors $w_{1}=M_{l} z_{1}$ and $w_{2}=V^{+} z_{1}$, we have that

\footnotetext{
${ }^{3}$ Such a statement was proven in Hautus (1983).
} 
$z_{1}=M_{l}^{+} w_{1}+V w_{2}$. System $\Phi$ in these new coordinates can be rewritten as follows:

$$
\begin{aligned}
\dot{w}_{1} & =\bar{A}_{1} w_{1}+\bar{D}_{1}\left(v-K^{*} w_{2}\right), \\
\dot{w}_{2} & =\bar{A}_{2} w_{1}+\bar{A}_{3} w_{2}+\bar{D}_{2}\left(v-K^{*} w_{2}\right), \\
\bar{y} & =\bar{C}_{1} w_{1}+\bar{F}\left(v-K^{*} w_{2}\right),
\end{aligned}
$$

where

$$
\begin{array}{ll}
\bar{A}_{1}=M_{l}\left(\bar{A}+\bar{D} K^{*} V^{+}\right) M_{l}^{+}, & \bar{D}_{1}=M_{l} \bar{D}, \\
\bar{A}_{2}=V^{+}\left(\bar{A}+\bar{D} K^{*} V^{+}\right) M_{l}^{+}, & \bar{D}_{2}=V^{+} \bar{D}, \\
\bar{A}_{3}=V^{+}\left(\bar{A}+\bar{D} K^{*} V^{+}\right) V, & \bar{C}_{1}=\bar{C} M_{l}^{+} .
\end{array}
$$

Thus, as for SD, it is known that system $\Phi$ is SD if, and only if, rank $\left[\begin{array}{c}\bar{D}_{1} \\ \bar{F}\end{array}\right]=\operatorname{rank}\left[\begin{array}{c}\bar{D} \\ \bar{F}\end{array}\right]$ and $\bar{A}_{3}$ is a Hurwitz matrix (see, e.g. Bejarano et al. (2009)).

Coming back to system $\Phi$. Define $\xi_{1}:=\left(\bar{F}^{\perp} \bar{C}\right)^{\perp \perp} \bar{F}^{\perp} \bar{y}=$ $M_{1} z_{1}$, with $M_{1}$ defined as in (11). Let us derive the vector $\xi_{1}$ :

$$
\dot{\xi}_{1}(t)=M_{1} \bar{A} z_{1}(t)+M_{1} \bar{D} v(t) .
$$

Let us define a new vector $\xi_{2}$ as follows

$$
\xi_{2}:=N_{2}^{\perp \perp} T_{1}\left[\begin{array}{c}
\dot{\xi}_{1} \\
\bar{y}(t)
\end{array}\right]
$$

with $N_{2}^{\perp \perp}$ and $T_{1}$ defined by (11). Thus, taking into account (7), (15), and (11), we have that

$$
\frac{d}{d t} J_{2}\left[\begin{array}{c}
\bar{y} \\
\int_{t_{0}}^{t} \bar{y}(\tau) d \tau
\end{array}\right]=\xi_{2}=M_{2} z_{1}(t), t>t_{0} \geq 0,
$$

where

$$
J_{2}=N_{2}^{\perp \perp} T_{1}\left[\begin{array}{cc}
J_{1} & 0 \\
0 & I_{\bar{p}}
\end{array}\right], \quad J_{1}=\left(\bar{F}^{\perp} \bar{C}\right)^{\perp \perp} \bar{F}^{\perp} .
$$

In the first identity of (17), we take outside the differential operator from (16) and use the definition of $\xi_{1}$. Thus, we can follow an iterative procedure to obtain the following set of equations, for $k \geq 1$,

$$
\frac{d^{k}}{d t^{k}} J_{k+1}\left[\begin{array}{c}
\bar{y}(t) \\
\vdots \\
\int_{t_{0}}^{t} \cdots \int_{t_{0}}^{\tau_{2}} \bar{y}\left(\tau_{1}\right) d \tau_{1} \cdots d \tau_{k}
\end{array}\right]=M_{k+1} z_{1}
$$

where $M_{k+1}$ is defined by (11), and $J_{k+1}$ is defined by the following recursive algorithm, for $k \geq 1$,

$$
J_{1}=\left(\bar{F}^{\perp} \bar{C}\right)^{\perp \perp} \bar{F}^{\perp}, \quad J_{k+1}=N_{k+1}^{\perp \perp} T_{k}\left[\begin{array}{cc}
J_{k} & 0 \\
0 & I_{\bar{p}}
\end{array}\right] .
$$

Thus $M_{k} z_{1}$ is expressed by a high order derivative of a function of $y(t)$. In such a way a real-time differentiator could be used, two of them frequently used due to their finite time convergence can be found in Levant (2003) and Mboup et al. (2009a). For instance if rank $M_{l}=\rho_{E}$, then $z_{1}$ is algebraically observable, i.e. it could be reconstructed by using a real-time differentiator.
In order to match system $\Sigma$ with system $\Phi$, from now on, we define $\bar{y}=\left[\begin{array}{ll}0_{1 \times n-\rho_{E}} & y^{T}\end{array}\right] \in \mathbb{R}^{\bar{p}}\left(\bar{p}:=n-\rho_{E}+p\right)$, and $v(t)=\left[\begin{array}{ll}z_{2}^{T}(t) & \mu^{T}(t)\end{array}\right]^{T} \in \mathbb{R}^{q}\left(q=n-\rho_{E}+m\right)$, then in view of (6), equations (5) and (7) are identical. Below, we consider two cases: when $\Sigma$ is SO and when it is SD, but not SO. Of course, since $\Phi$ is a standard linear system, there might be other methods, besides the one proposed below, that might be used to carry out the algebraic reconstruction of the state.

\subsection{Finite time reconstruction}

Let us consider that system $\Sigma$ is SO. Then, the reconstruction of entire state vector $x(t)$ can be achieved in finite time: by means of an algebraic formula. Let us proceed in the following way. Since $\Phi$ is SO, $\rho_{M_{l}}=\rho_{E}$. Then in this case, from (18), we obtain the equation

$$
\frac{d^{l-1}}{d t^{l-1}} M_{l}^{-1} J_{l}\left[\begin{array}{c}
\bar{y}(t) \\
\vdots \\
\int_{t_{0}}^{t} \cdots \int_{t_{0}}^{\tau_{2}} \bar{y}\left(\tau_{1}\right) d \tau_{1} \cdots d \tau_{l-1}
\end{array}\right]=z_{1}
$$

where $M_{l} \in \mathbb{R}^{\rho_{E} \times \rho_{E}}$ and $J_{l} \in \mathbb{R}^{\rho_{E} \times \bar{p} l}$. Let $U$ be a matrix so that

$$
\operatorname{rank}\left[\begin{array}{c}
D \\
F
\end{array}\right] U=\operatorname{rank}\left[\begin{array}{c}
D \\
F
\end{array}\right]=: \bar{m}, \quad U \in \mathbb{R}^{q \times \bar{m}}
$$

Since (9) must be satisfied according to Theorem 1, we have that

$z_{2}(t)=\left[\begin{array}{cc}I_{n-\rho_{E}} & 0_{\bar{q}}\end{array}\right]\left(\left[\begin{array}{c}\bar{D} \\ \bar{F}\end{array}\right] \begin{array}{cc}I & 0 \\ 0 & U\end{array}\right)^{+}\left(\left[\begin{array}{c}\dot{z}_{1}(t) \\ \bar{y}\end{array}\right]-\left[\begin{array}{c}\bar{A} \\ \bar{C}\end{array}\right] z_{1}(t)\right)$

where $\bar{q}:=n-\rho_{E}+\bar{m}$. Now, we are ready to give a formula to reconstruct $x$ in finite time.

Theorem 3. If system $\Sigma$ is $S O$, then the state $x$ can be expressed algebraically by the following formula:

$$
x(t)=\frac{d^{l}}{d t^{l}}\left[\begin{array}{ll}
S & 0_{n \times \bar{m}}
\end{array}\right]\left[\begin{array}{c}
H_{1} \\
H_{2}
\end{array}\right]\left[\begin{array}{c}
\bar{y}(t) \\
\vdots \\
\int_{t_{0}}^{t} \cdots \int_{t_{0}}^{\tau_{1}} \bar{y}\left(\tau_{1}\right) d \tau_{1} \cdots d \tau_{l}
\end{array}\right],
$$

where $H_{1} \in \mathbb{R}^{\rho_{E} \times \bar{p}(l+1)}$ and $H_{2} \in \mathbb{R}^{\bar{q} \times \bar{p}(l+1)}$ are defined as

$$
\begin{aligned}
H_{1} & :=\left[\begin{array}{cc}
0_{\rho_{E} \times \bar{p}} & M_{l}^{-1} J_{l}
\end{array}\right], H_{2}:=\left[\begin{array}{c}
\bar{D} U \\
\bar{F} U
\end{array}\right]^{+}\left(G_{1}-G_{2}\right), \\
G_{1} & :=\left[\begin{array}{cc}
M_{l}^{-1} J_{l} & 0_{\rho_{E} \times \bar{p}} \\
0_{\bar{p} \times \bar{p} l} & I_{\bar{p} \times \bar{p}}
\end{array}\right], G_{2}:=\left[\begin{array}{cc}
0_{\rho_{E} \times \bar{p}} & \bar{A} M_{l}^{-1} J_{l} \\
0_{\bar{p} \times \bar{p}} & \bar{C} M_{l}^{-1} J_{l}
\end{array}\right],
\end{aligned}
$$

$G_{1}, G_{2} \in \mathbb{R}^{\rho_{E}+\bar{p} \times \bar{p}(l+1)}$, and $M_{l}$ and $J$ defined recursively in (11) and (19), respectively, and $U$ defined by (21).

Proof. Let us define the extended vector $Y_{k} \in \mathbb{R}^{\bar{p}(k+1)}$ as follows

$$
Y_{k}=\left[\begin{array}{c}
\bar{y}(t) \\
\int_{t_{0}}^{t} \bar{y}\left(\tau_{1}\right) d \tau_{1} \\
\vdots \\
\int_{t_{0}}^{t} \cdots \int_{t_{0}}^{\tau_{1}} \bar{y}\left(\tau_{1}\right) d \tau_{1} \cdots d \tau_{k}
\end{array}\right], k=1,2, \ldots
$$


Then for a matrix $X$ of suitable dimension the following holds:

$$
\frac{d^{k}}{d t^{k}} X Y_{k}=\frac{d^{k+1}}{d t^{k+1}}\left[\begin{array}{ll}
0 & X
\end{array}\right] Y_{k+1}
$$

Thus, since $x=S z$, by manipulating (22) and tanking into account (20), (23) is obtained.

Remark 1. One might obtain a little more from the previous analysis, that is, one can express by an algebraic formula the part of $\mu$ that can be reconstructed (assuming $\Sigma$ is SO). Indeed, let $\bar{\mu}$ be implicitly defined by the equation $\left[\begin{array}{cc}D^{T} & F^{T}\end{array}\right]^{T} \mu=$ $\left[\begin{array}{cc}D^{T} & F^{T}\end{array}\right]^{T} U \bar{\mu}$. With the same procedure followed to obtain (23), $\bar{\mu}$ can be expressed by the formula

$\bar{\mu}(t)=\frac{d^{l}}{d t^{l}}\left[\begin{array}{ll}0_{\bar{m} \times n} & I_{\bar{m}}\end{array}\right]\left[\begin{array}{c}H_{1} \\ H_{2}\end{array}\right]\left[\begin{array}{c}\bar{y}(t) \\ \vdots \\ \int_{t_{0}}^{t} \cdots \int_{t_{0}}^{\tau_{1}} \bar{y}\left(\tau_{1}\right) d \tau_{1} \cdots d \tau_{l}\end{array}\right]$.

\subsection{Asymptotic Reconstruction}

Let us assume that $\Sigma$ is SD, but not SO. Next we show how to carry out the estimation of $x(t)$.

Theorem 4. Assuming that $\Sigma$ is $S D$, but not $S O$, we obtain that

$$
\lim _{t \rightarrow \infty}\|x(t)-\hat{x}(t)\|=0
$$

provided that $\hat{x}(t)$ is designed by following (26)-(27).

$$
\begin{gathered}
\hat{x}(t)=\frac{d^{l}}{d t^{l}}\left(\left[\begin{array}{ll}
S & 0_{n \times \bar{m}}
\end{array}\right]\left[\begin{array}{c}
\bar{H}_{1} \\
\bar{H}_{2}
\end{array}\right] Y_{l}(t)\right)\left[\begin{array}{ll}
S & 0_{n \times m}
\end{array}\right]\left[\begin{array}{c}
V \\
K^{*}
\end{array}\right]_{(26)}^{\hat{w}_{2}} \\
\dot{\hat{w}}_{2}=\bar{A}_{3} \hat{w}_{2}+\bar{A}_{2} \frac{d^{l}}{d t^{l}}\left(\left[\begin{array}{ll}
0_{\rho_{E}-\rho_{M} \times \bar{p}} & J_{l}
\end{array}\right]+\bar{D}_{2} U \bar{H}_{2}\right) Y_{l}
\end{gathered}
$$

where $\bar{H}_{1} \in \mathbb{R}^{\rho_{E} \times \bar{p}(l+1)}, \bar{H}_{2} \in \mathbb{R}^{\bar{q}+\bar{p} \times \bar{p}(l+1)}$, and $\bar{G}_{1}, \bar{G}_{2} \in$ $\mathbb{R}^{\rho_{M}+\bar{p} \times \bar{p}(l+1)}\left(\rho_{M}=\rho_{M_{l}}\right)$ satisfy the following identities,

$$
\begin{gathered}
\bar{H}_{1}=\left[\begin{array}{cc}
0_{\rho_{E} \times \bar{p}} & M_{l}^{+} J_{l}
\end{array}\right], \bar{H}_{2}:=\left[\begin{array}{c}
\bar{D}_{1} U \\
\bar{F} U
\end{array}\right]^{+}\left(\bar{G}_{1}-\bar{G}_{2}\right), \\
\bar{G}_{1}:=\left[\begin{array}{cc}
J_{l} & 0_{\rho_{M} \times \bar{p}} \\
0_{\bar{p} \times \bar{p} l} & I_{\bar{p}}
\end{array}\right], G_{2}:=\left[\begin{array}{cc}
0_{\rho_{M} \times \bar{p}} & \bar{A}_{1} J_{l} \\
0_{\bar{p} \times \bar{p}} & \bar{C}_{1} J_{l}
\end{array}\right] .
\end{gathered}
$$

Proof. In this case, as $\Sigma$ is not SO, by differentiation, we are able to reconstruct $w_{1}=M_{l} z_{1}$ only, where $\rho_{M_{l}}<\rho_{E}$. Since $\Phi$ is SD and by (21), we have that $\left[\begin{array}{c}M_{l} \bar{D} \\ \bar{F}\end{array}\right] U$ has full column rank. Then, from (18) and from (13a) and (13c), we have the following expression for $w_{1}$ and $z_{2}$,

$$
w_{1}(t)=\frac{d^{l-1}}{d t^{l-1}} J_{l} Y_{l-1}(t),
$$

$z_{2}(t)=\frac{d^{l}}{d t^{l}}\left[\begin{array}{ll}I_{n-\rho_{E}} & 0_{n-\rho_{E} \times \bar{m}}\end{array}\right] \bar{H}_{2} Y_{l}(t)+\left[\begin{array}{ll}I_{n-\rho_{E}} & 0_{n-\rho_{E} \times m}\end{array}\right] K^{*} w_{2}$.
Thus, since $x=S z$ and $z_{1}=M_{l}^{+} w_{1}+V w_{2}$, we obtain the identity $x(t)=\frac{d^{l}}{d t^{l}}\left(\left[\begin{array}{ll}S & 0_{n \times \bar{m}}\end{array}\right]\left[\begin{array}{c}\bar{H}_{1} \\ \bar{H}_{2}\end{array}\right] Y_{l}(t)\right)+\left[\begin{array}{cc}S & 0_{n \times m}\end{array}\right]\left[\begin{array}{c}V \\ K^{*}\end{array}\right] w_{2}$.

Therefore, by comparing (26) with (30), we have that

$$
\hat{x}(t)=x(t)-\left[\begin{array}{cc}
S & 0_{n \times \bar{m}}
\end{array}\right]\left[\begin{array}{c}
V \\
K^{*}
\end{array}\right]\left(w(t)-\hat{w}_{2}(t)\right) .
$$

Furthermore, in view of (13b), (28), and (27), we obtain that

$$
\dot{w}_{2}-\dot{\hat{w}}_{2}=\bar{A}_{3}\left(w_{2}-\hat{w}_{2}\right) \text {. }
$$

Since $\Phi$ is $\mathrm{SD}, \bar{A}_{3}$ is Hurwitz; hence $\hat{w}_{2}$ converges to $w_{2}$. Then by (31) the proof is finished.

Remark 2. If $\bar{\mu}$ needs to be reconstructed also, then it can be done by means of $\widehat{\bar{\mu}}(t)$, defined as follows,

$\widehat{\bar{\mu}}(t)=\frac{d^{l}}{d t^{l}}\left(\left[\begin{array}{ll}0_{\bar{m} \times n} & I_{\bar{m}}\end{array}\right]\left[\begin{array}{c}\bar{H}_{1} \\ \bar{H}_{2}\end{array}\right] Y_{l}(t)\right)\left[\begin{array}{ll}0_{\bar{m} \times n} & I_{\bar{m}}\end{array}\right]\left[\begin{array}{c}V \\ \bar{K}^{*}\end{array}\right] \hat{w}(t)$

where $\bar{K}^{*}$ is implicitly defined by the equation

$$
\left[\begin{array}{c}
\bar{D} \\
\bar{F}
\end{array}\right] K^{*}=\left[\begin{array}{c}
\bar{D} \\
\bar{F}
\end{array}\right]\left[\begin{array}{cc}
I_{n-\rho_{E}} & 0 \\
0 & U
\end{array}\right] \bar{K}^{*}
$$

So, we obtain straightforwardly that $\|\hat{\mu}(t)-\mu(t)\|$ goes to zero.

\subsection{Summarized algorithm for the reconstruction of $x(t)$}

Below, there is a step-by-step description that may be followed to carry out the estimation of $x(t)$.

Step 1. Choose $S$ and $T$ to bring $E$ into the form (4).

Step 2. Calculate matrices $\bar{A}, \bar{C}, \bar{D}$, and $\bar{F}$ according to (8).

Step 3. Calculate matrices $M_{l}$ and $J_{l}$ following the recursive algorithms (11) and (19), respectively. Let us remind that $l$ is such that $\rho_{M_{l+1}}=\rho_{M_{l}}>\rho_{M_{l-1}}$.

Step 4A. Check if $\Sigma$ is SO: test both conditions i) $\rho_{M_{l}}=\rho_{E}$ and ii) condition in (9). If they both are satisfied go to Step 5.A.

Step 4B. Check if $\Sigma$ is SD: test the conditions i) $\operatorname{rank}\left[\begin{array}{c}\bar{D}_{1} \\ \bar{F}\end{array}\right]=$ $n-\rho_{E}+\operatorname{rank}\left[\begin{array}{c}D \\ F\end{array}\right]$ and ii) $\bar{A}_{3}$ is Hurwitz $\left(\bar{D}_{1}\right.$ and $\bar{A}_{3}$ defined in (14)). If they both are satisfied, go to Step 5.B. Otherwise, the exact estimation (even asymptotic) of $x$ is impossible.

Step 5A. $x(t)$ is reconstructed by means of the formula (23).

Step 5B. $x(t)$ is estimated by using $\hat{x}(t)$ described in (26)-(27).

To carry out Step 5 one needs to use a real-time differentiator. In the example presented in the next section, we use algebraic numerical and sliding mode differentiators, which have been proposed in the last years and have a considerable acceptation in several observation and identification procedures thanks to their finite time convergence and robustness. A brief review of those differentiators is given in Appendix A. 


\section{Example}

Let us consider that $\Sigma$ has the following matrices values

$$
\begin{gathered}
E=\left[\begin{array}{llll}
0 & 1 & 0 & 1 \\
0 & 0 & 0 & 0 \\
1 & 0 & 1 & 1 \\
0 & 0 & 0 & 0
\end{array}\right], A=\left[\begin{array}{cccc}
-1 & -1 & 1 & 1 \\
2 & 1 & 2 & 0 \\
1 & 1 & 1 & -1 \\
0 & 0 & 0 & 0
\end{array}\right], D=\left[\begin{array}{c}
1 \\
-1 \\
0 \\
0
\end{array}\right] \\
C=\left[\begin{array}{cccc}
0 & 1 & 0 & 1 \\
0 & -1 & 0 & 2
\end{array}\right], F=\left[\begin{array}{l}
0 \\
1
\end{array}\right] .
\end{gathered}
$$

It is easy to see that, in this example, $\operatorname{det}(s E-A)=0$ for every $s \in \mathbb{C}$. Hence, many solutions for $x(t)$ are expected to satisfy the differential equation in (1). However, to each output $y(t)$ corresponds only one trajectory of $x(t)$ (a.e.). Indeed, we will see that, according to Theorem $1, \Sigma$ is SO. We describe the observer design following the step by step description.

Step 1. For this case matrices $S$ and $T$ are chosen as follows,

$$
S=\left[\begin{array}{cccc}
0 & 0 & -1 & -1 \\
0 & 0 & 0 & -1 \\
0 & 1 & 1 & 0 \\
1 & 0 & 0 & 1
\end{array}\right], \quad T=\left[\begin{array}{cccc}
1 & 0 & 0 & 0 \\
-1 & 0 & 1 & 0 \\
0 & 1 & 0 & 0 \\
0 & 0 & 0 & 1
\end{array}\right]
$$

Step 2. Matrices $\bar{A}, \bar{C}, \bar{D}$, and $\bar{F}$ take the following values:

$$
\begin{aligned}
& \bar{A}=\left[\begin{array}{cc}
1 & 1 \\
-2 & 0
\end{array}\right], \quad \bar{D}=\left[\begin{array}{ccc}
2 & 3 & 1 \\
-2 & -6 & -1
\end{array}\right], \\
& \bar{C}=\left[\begin{array}{ll}
0 & 2 \\
0 & 0 \\
1 & 0 \\
2 & 0
\end{array}\right], \quad \bar{F}=\left[\begin{array}{ccc}
0 & -3 & -1 \\
0 & 0 & 0 \\
0 & 0 & 0 \\
0 & 3 & 1
\end{array}\right] .
\end{aligned}
$$

Step 3. Matrices $M_{2}$ and $J_{2}$ take the values

$$
\begin{aligned}
M_{2} & =\left[\begin{array}{cc}
1 & 0 \\
\sqrt{2} & \sqrt{2}
\end{array}\right], \\
J_{2} & =\left[\begin{array}{cccccccc}
0 & 0 & 0 & 0 & 0 & 0 & 1 & 0 \\
0 & 0 & 0 & 0 & \sqrt{2} / 2 & 0 & 0 & \sqrt{2} / 2
\end{array}\right] .
\end{aligned}
$$

Step 4. Here, $\operatorname{rank} M_{2}=2, \operatorname{rank} \bar{B}=3$, and $n-\rho_{E}=2$. Therefore, both conditions of Theorem 1 are satisfied.

Step 5. The reconstruction of $x(t)$ can be done by means of the formula (23), once we define $\bar{y}^{T}=\left[\begin{array}{ccc}0 & 0 & y^{T}\end{array}\right]$. For this example, the reconstruction of $\mu$ is also possible following formula (25). Thus, we have

$$
\begin{aligned}
x_{1} & =-\frac{1}{3} y_{1}+\frac{7}{12} y_{2}-\frac{1}{2} \dot{y}_{1}+\frac{1}{6} \dot{y}_{2}, \\
x_{2} & =\frac{2}{3} y_{1}-\frac{1}{6} y_{2}+\frac{1}{6} \dot{y}_{2}, \\
x_{3} & =-\frac{1}{4} y_{2}+\frac{1}{2} \dot{y}_{1}, \\
x_{4} & =\frac{1}{3} y_{1}-\frac{1}{6} y_{2}+\frac{1}{6} \dot{y}_{2}, \\
\mu & =\frac{1}{2} y_{2}+\frac{1}{2} \dot{y}_{2} .
\end{aligned}
$$

To estimate the state $x$ and $\mu$, we use two different differentiators, an algebraic numerical differentiator (ALND) and a high order sliding mode differentiator (HOSMD).

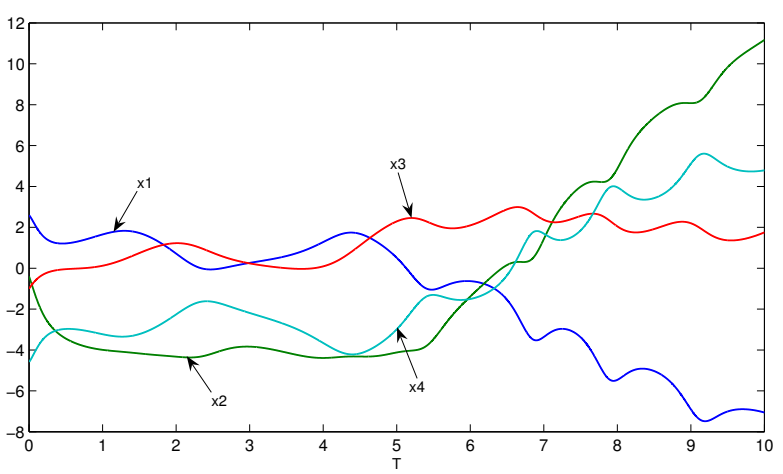

Figure 1: Trajectories of $x(t)$.

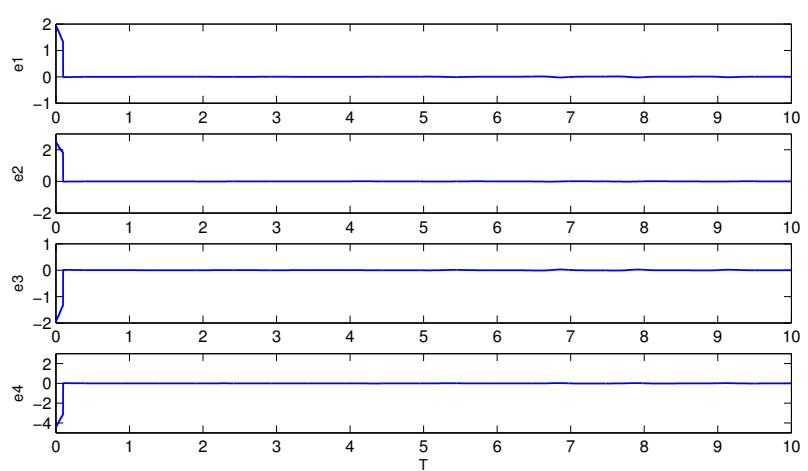

Figure 2: Estimation error $e=x-\hat{x}$ using ALND.

For simulation purposes, we have chosen $\mu=$ $2 \sin \left(x_{1}-x_{2}+x_{3}\right)+\cos (t)$ and $x_{1}=x_{2}-2-\cos (3 t)$. The state trajectories are depicted in Figure 1. The estimation error is depicted in Figures 2 and 3. The corresponding estimation for $\mu$, denoted by $\hat{\mu}$, is shown in Figure 4 . We have also tested both differentiators in presence of output noise, for that we have used an approximation of white noise using the Matlab command rand with a uniform distribution on the interval $(-0.1,0.1)$ for the first output and $(-0.05,0.05)$ for the second one. The estimation error is depicted in Figure 5.

\section{Conclusions}

We have given necessary and sufficient conditions to estimate the slow (non-impulsive) trajectories, for singular systems

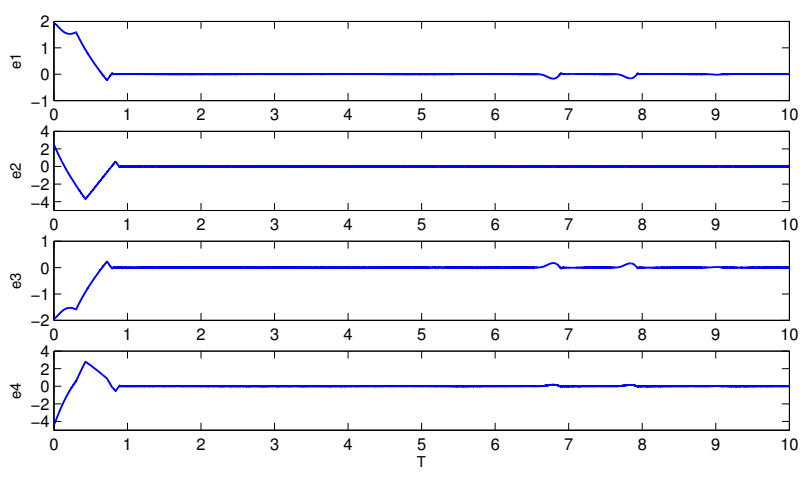

Figure 3: Estimation error $e=x-\hat{x}$ using HOSMD. 


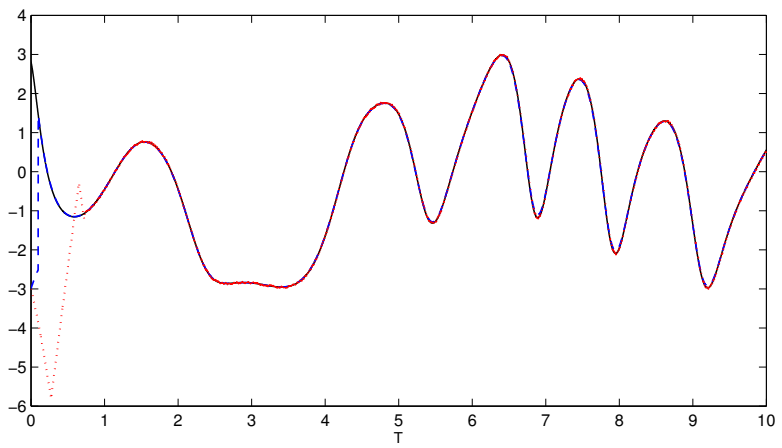

Figure 4: UI $\mu$ (solid) and its estimation $\hat{\mu}$ (dashed for ALND, dotted HOSMD).

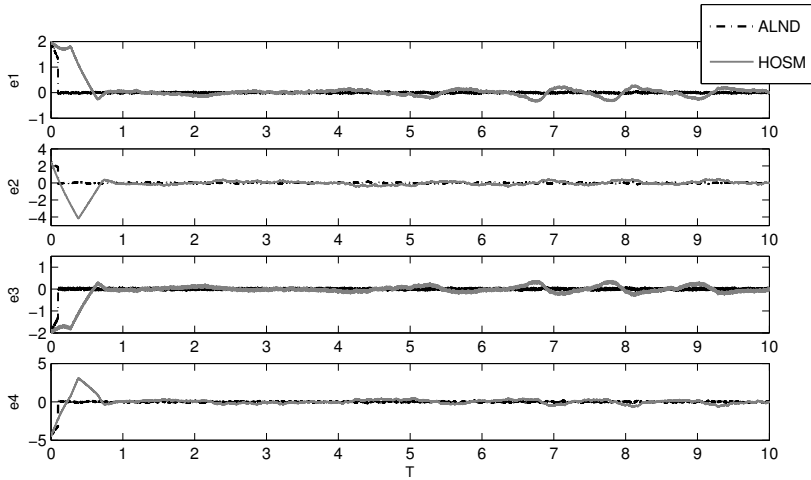

Figure 5: Estimation error $e=x-\hat{x}$ with a noisy output.

in which more than one solution of the differential equation is allowed, i.e. the pencil of the system is not required to be regular as it is assumed in most of the previous works where the observability is studied. We have given explicit formulas to reconstruct in finite time and asymptotically the states. Nevertheless, we have to notice that when the estimate of $x$ needs an excessive number of derivatives, the error due to noise and sampling time could increase considerably. In that case, if an asymptotic estimation is enough, we should use the on-line differentiation only the needed times allowing after to design a Luenberger-like observer. In that case, a simple but cumbersome modified procedure might be followed in order to reduce at minimum the number of derivatives required to estimate the state, we refer the interested reader to Floquet et al. (2007) and Bejarano and Fridman (2010).

\section{Acknowledgement}

This research has been financially supported by EU Interreg IV A 2 Mers Seas Zeeën Cross-border Cooperation Programme under SYSIASS project 06-020. For more details about the SYSIASS project see the project website www.sysiass.eu. F.J. Bejarano held a posdoctoral position at INRIA - Nord Europe with the ALIEN team, from October 2010 to September 2011, period during which this work was produced.

\section{Appendix A. Review of two differentiators}

\section{Appendix A.1. ALND}

This algebraic setting for numerical differentiation of noisy signals is introduced in Fliess et al. (2004) and analyzed in Liu et al. (2011a,b); Mboup et al. (2007, 2009b) (see also Nöthen (2007) for interesting discussions and comparisons). The reader may find additional theoretical foundations in Fliess (2006); Fliess and Sira-Ramírez (2003). Consider a signal $y(t)=$ $\sum_{l=0}^{\infty} y^{(l)}(0) \frac{t^{l}}{l !}$ which is assumed to be analytic around $t=0$ and its truncated Taylor expansion $y_{N}(t)=\sum_{l=0}^{N} y^{(l)}(0) \frac{t^{l}}{l !}$ at order $N$. The usual rules of symbolic calculus in Schwartz's distribution theory (Schwartz (1966)) yield $y_{N}^{(N+1)}(t)=y(0) \delta^{(N)}+\ldots+$ $y^{(N)}(0) \delta$, where $\delta$ is the Dirac measure at zero. Multiply both sides by $(-t)^{l}:(-t)^{l} y_{N}^{(N+1)}(t)=(-t)^{l}\left(y(0) \delta^{(N)}+\ldots+y^{(N)}(0) \delta\right)$, and apply the rules $t \delta=0, t \delta^{(l)}=-l \delta^{(l-1)}, l \geq 1$. We obtain a triangular system of linear equations from which the derivatives $y^{(l)}(0)$ can be obtained $(1 \leq l \leq N)$

$$
(-t)^{l} y_{N}^{(N+1)}(t)=\frac{N !}{(N-l) !} \delta^{(N-l)} y(0)+\ldots+\delta y^{(N-l)}(0) .
$$

It means that the coefficients $y(0), \ldots, y^{(N)}(0)$ are linearly identifiable Fliess and Sira-Ramírez (2003); Fliess and SiraRamirez (2008). The time derivatives of $y_{N}(t)$, the Dirac measures and its derivatives are removed by integrating with respect to time both sides of Eq. (A.1) at least $v$ times $(v>N)$ :

$$
\begin{aligned}
\int_{0}^{t} & \int_{0}^{t_{\nu-1}} \cdots \int_{0}^{t_{1}}(-\tau)^{l^{2}} y_{N}^{(N+1)} d t_{v-1} \cdots d t_{1} d \tau= \\
& \frac{N !}{(N-l) !} \frac{t^{\nu-N-l-1}}{(v-N-l-1) !} y(0)+\ldots+\frac{t^{\nu-1}}{(v-1) !} y^{(N-l)}(0) .
\end{aligned}
$$

The iterated integrals may be replaced by

$\int_{0}^{t} \int_{0}^{t_{\nu-1}} \cdots \int_{0}^{t_{1}} \tau^{\alpha} x(\tau) d t_{v-1} \cdots d t_{1} d \tau=\int_{0}^{t} \frac{(t-\tau)^{\nu-1}}{(v-1) !} \tau^{\alpha} x(\tau) d \tau$.

It is clear that the numerical estimation rely on $\lim _{N \rightarrow+\infty}\left[y_{N}^{(l)}(0)\right]_{\mathrm{estim}}(t)=y^{(l)}(0)$.

Remark 3. These iterated integrals are low pass filters which attenuate the noises, which are viewed as highly fluctuating phenomena (see Fliess (2006) for more details). The above formulae may easily be extended to sliding time windows in order to obtain real time estimates (see Mboup et al. (2007, 2009b); Liu et al. (2011a,b) for further details). Moreover, according to the performed algebraic manipulation one can have some different formulae: $\forall t_{0} \in I$,

$\tilde{x}_{t_{0} \pm}^{(n)}(k, \mu, \beta T)=\frac{1}{(\beta T)^{n}} \int_{0}^{1} \gamma_{k, \mu, n}(1-\tau)^{\mu} \tau^{k} P_{n}^{\mu, k}(\tau) x\left(t_{0}+\beta T \tau\right) d \tau$,

where $x$ is assumed to be analytic, $P_{n}^{\mu, k}$ is the $n^{\text {th }}$ order Jacobi polynomial defined on $[0,1]$ with $n \in N, k, \mu \in]-1, \infty[, \beta= \pm 1$ and $T>0$.

\section{Appendix A.2. HOSMD}

The HOSMD described in Levant (2003) can be expressed in a dynamic form by means of the equations (A.4), where the 
signal to be differentiated is represented by a function $f \in C^{r+1}$.

$$
\begin{aligned}
\dot{\alpha}_{0} & =-\lambda_{r} L^{\frac{1}{r+1}}\left|\alpha_{0}-f\right|^{\frac{r}{r+1}} \operatorname{sign}\left(\alpha_{0}-f\right)+\alpha_{1} \\
\dot{\alpha}_{1} & =-\lambda_{r-1} L^{\frac{1}{r}}\left|\alpha_{1}-\dot{\alpha}_{0}\right|^{\frac{r-1}{r}} \operatorname{sign}\left(\alpha_{1}-\dot{\alpha}_{0}\right)+\alpha_{2} \\
\vdots & \\
\dot{\alpha}_{r-1} & =-\lambda_{1} L^{\frac{1}{2}}|\cdot|^{\frac{1}{2}} \operatorname{sign}\left(\alpha_{r-1}-\dot{\alpha}_{r-2}\right)+\alpha_{r} \\
\dot{\alpha}_{r} & =-\lambda_{0} L \operatorname{sign}\left(\alpha_{r}-\dot{\alpha}_{r-1}\right)
\end{aligned}
$$

Then, in the cited paper it was shown that if $\lambda_{i}{ }^{\prime} s$ gains are chosen properly, the differentiator converges in a finite time $T$, i.e., $\alpha_{i}(t)=f^{(i)}(t)$, for all $t \geq T$ and $i=0,1, \ldots, r$. $L$ is a constant such that $\left\|f^{(r+1)}(t)\right\| \leq L$. For the case when $r=5$, the gains could be chosen as $\lambda_{0}=1.1, \lambda_{1}=1.5, \lambda_{2}=3, \lambda_{3}=5, \lambda_{4}=8$, $\lambda_{5}=12$.

\section{References}

Bejarano, F. J., Fridman, L., 2010. High order sliding mode observer for linear systems with unbounded unknown inputs. International Journal of Control 83 (9), 1920-929.

Bejarano, F. J., Fridman, L., Poznyak, A., 2009. Unknown input and state estimation for unobservable systems. SIAM J. Control Optim. 48 (2), 11551178 .

Boukhobza, T., Hamelin, F., July 2007. State and input observability for structured linear systems: A graph-theoretic approach. Automatica 43 (7), 1204 1210 .

Chu, D., Mehrmann, V., 1999. Disturbance decoupled observer design for descriptor systems. Systems \& Control Letters 38, 37-48.

Cobb, D., 1984. Controllability, observability, and duality in singular systems. IEEE Transactions on Automatic Control AC-29 (12), 1076-1082.

Darouach, M., Boutat-Baddas, L., 2008. Observers for a class of nonlinear singular systems. IEEE Transactions on Automatic Control 53 (11), 26272633.

Darouach, M., Boutayeb, M., July 1995. Design of observers for descriptor systems. IEEE Transactions on Automatic Control 40 (7), 1323-1327.

Darouach, M., Zasadzinski, M., Hayar, M., 1996. Reduced-order observer design for descriptor systems with unknown inputs. IEEE Transactions on Automatic Control 41 (7), 1068-1072.

Darouach, M., Zasadzinski, M., Xu, S. J., 1994. Full-order observers for linear systems with unknown inputs. IEEE Transactions on Automatic Control 39 (3), 606-609.

Diop, S., Fliess, M., 1991. Nonlinear observability, identifiability, and persistent trajectories. In: Prcoceedings of the 30th Conference on Decision and Control. Brighton, England, pp. 714-719.

Fliess, M., 2006. Analyse non standard du bruit. C.R. Acad. Sci. Paris, ser. I $342,797-802$.

Fliess, M., Join, C., Mboup, M., Sira-Ramírez, H., 2004. Compression différentielle de transitoires bruités. C.R. Acad. Sci. Paris, ser. I 339, 821826.

Fliess, M., Sira-Ramírez, H., 2003. An algebraic framework for linear identification. ESAIM Control Optim. Calc. Variat. 9, 151-168.

Fliess, M., Sira-Ramirez, H., 2008. Closed-loop parametric identification for continuous-time linear systems via new algebraic techniques. In: Wang, H. G. . L. (Ed.), Identification of Continuous-time Models from Sampled Data. Advances in Industrial Control. Springer, pp. 362-391.

URL http: //hal .inria.fr/inria-00114958/en/

Floquet, T., Barbot, J., December 2004. A sliding mode approach of unknown input observers for linear systems. In: 43rd IEEE Conference on Decision and Control. Atlantis, Paradise Island, Bahamas, pp. 1724-1729.

Floquet, T., Edwards, C., Spurgeon, S., 2007. On sliding mode observers for systems with unknown inputs. Int. J. Adapt. Control Signal Process 21 (89), 638-656.

Guan, Y., Saif, M., 1992. A novel approach to the design of unknown input observers. IEEE Transactions on Automatic Control 36 (5), 871-875.

Hautus, M., April 1983. Strong detectability and observers. Linear Algebra and its Applications 50, 353-368.
Hou, M., Müller, P., 1999a. Casual observability of descriptor systems. IEEE Transactions on Automatic Control 44 (1), 158-163.

Hou, M., Müller, P., 1999b. Observer design for descriptor systems. IEEE Transactions on Automatic Control 44 (1), 164-168.

Kaczorek, T., 2007. Polynomial and Rational Matrices Applications in Dynamical Systems Theory. Communications and control engineering. Springer.

Koenig, D., 2005. Unknown input proportional multiple-integral oberver design for linear descriptor systems: application to state and fault estimation. IEEE Transactions on Automatic Control 50 (2), 212-217.

Levant, A., 2003. High-order sliding modes: differentiation and outputfeedback control. International Journal of Control 76 (9-10), 924-941.

Liu, D.-Y., Gibaru, O., Perruquetti, W., 2011a. Differentiation by integration with Jacobi polynomials. Journal of Computational and Applied Mathematics, 21 pages.

URL http://hal .inria.fr/inria-00550160/en/

Liu, D.-Y., Gibaru, O., Perruquetti, W., Mar. 2011b. Error analysis of Jacobi derivative estimators for noisy signals. Numerical Algorithms.

URL http: //hal .inria.fr/inria-00573270/en/

Mboup, M., Join, C., Fliess, M., July 2007. A revised look at numerical differentiation with an application to nonlinear feedback control. In: Proceedings of the 15th Mediterranean Conference on Control \& Automation. Athens, Greece, pp. 1-6.

Mboup, M., Join, C., Fliess, M., 2009a. Numerical differentiation with annihilators in noisy environment. Numerical Algorithms 50 (4), 439-467.

Mboup, M., Join, C., Fliess, M., 2009b. Numerical differentiation with annihilators in noisy environment. Numerical Algorithms 50 (4), 439-467.

Molinari, B. P., October 1976. A strong controllability and observability in linear multivariable control. IEEE Transactions on Automatic Control 21 (5), 761-764.

Nöthen, C., 2007. Beiträge zur rekonstruktion nicht direkt gemessener größen bei der silizium-einkristallzüchtung nach dem czochralski-verfahren. Diplomarbeit, Technische Universität Dresden.

Paraskevopoulos, P., Koumboulis, F., August 1992. Observers for singular systems. IEEE Transactions on Automatic Control 37 (8), 1211-1215.

Paraskevopoulos, P., Koumboulis, F., Tzierakis, K., Panagiotakis, G., April 1992. Observer design for generalized state space systems with unknown inputs. Systems \& Control Letters 18 (4), 309-321.

Schwartz, L., 1966. Théorie des distributions. Hermann.

Trentelman, H. L., Stoorvogel, A. A., Hautus, M. L. J., 2001. Control theory for linear systems. Communications and control engineering. Springer, New York, London.

Yip, E., Sincovec, R., 1981. Solvability, controllability, and observability of continuous descriptor systems. IEEE Transactions on Automatic Control AC-26 (3), 702-707. 